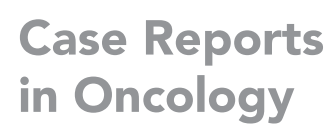

Case Reports

\title{
Tumor Lysis Syndrome in a Low-Risk Pancreatic Cancer Patient
}

\author{
Paul Travers Alexandra Goodman Bernard Poiesz \\ Internal Medicine, SUNY Upstate Medical University, Syracuse, NY, USA
}

\author{
Keywords \\ Tumor lysis · Risk stratification · Malignancy
}

\begin{abstract}
Tumor lysis syndrome (TLS) is the most common hematologic emergency encountered during the treatment of high-grade malignancies. While it can lead to death, the prognosis is typically excellent if caught early on in the course. Risk stratification prior to treatment initiation is paramount in deciding the utility of prophylaxis and ultimately in reducing morbidity and mortality. The following case describes the development of TLS in a patient categorized as low risk and highlights the need for further elucidation of a unified risk stratification system.
\end{abstract}

\section{Introduction}

Tumor lysis syndrome (TLS) is a hematologic emergency most commonly encountered during the treatment of high-grade hematologic malignancies [1]. It is rarely seen in cases of solid tumors; however, as therapies continue to become more effective and more targeted, its frequency in these cases has been increasing $[2,3]$. The syndrome occurs following rapid lysis of tumor cells causing release of intracellular contents into the bloodstream and can be classified as either laboratory or clinical via the Cairo and Bishop classification system [4]. Laboratory TLS is defined as the development of 2 of the following 4 laboratory abnormalities, in the same 24-h period, within 3 days prior to starting treatment and within 7 days after initiation of chemotherapy: hyperuricemia, hyperkalemia, hyperphosphatemia, and hypocalcemia. Clinical TLS is defined as the presence of laboratory TLS plus acute kidney injury, cardiac arrhythmia, or symptomatic hypocalcemia (seizure and tetany). We present a rapidly fatal case of clinical TLS in a patient with stage IV pancreatic adenocarcinoma following palliative treatment with gemcitabine and nab-paclitaxel.

\section{Karger $\stackrel{\text { ! }}{=}$}






Fig. 1. CT abdomen with contrast. Red arrows highlight one of many liver lesions found prior to initiating chemotherapy. Yellow arrow points to stranding and thickening of the small bowel mesentery. Green arrows point to nodular thickening and enhancement of peritoneal reflections.

\section{Case Presentation}

A 76-year-old female with a past medical history of stage III renal cell carcinoma status after right nephrectomy which did not require adjuvant treatment presented for follow-up to an outpatient clinic when she was found to have severe abdominal pain, tachycardia, and pallor. She had recently been diagnosed with pancreatic adenocarcinoma with metastatic disease to the liver complicated by splenic vein thrombosis. Upon admission to the hospital, she was found to have a leukocytosis count of $21,000 \mu \mathrm{L}$ and was started on broad-spectrum antibiotics for concern of spontaneous bacterial peritonitis. Creatinine was found to be mildly elevated at 1.10 $\mathrm{mg} / \mathrm{dL}$, so she was started on maintenance fluids. Further laboratory tests were within normal limits. CT abdomen/pelvis was obtained which showed re-demonstration of the liver metastasis and peritoneal stranding, which was concerning for peritoneal carcinomatosis (Fig. 1). A family meeting was held, and the decision was made to move forward with palliative chemotherapy with gemcitabine and nab-paclitaxel.

Overnight following her first dose of chemotherapy, her potassium increased to $6.1 \mathrm{mmol} / \mathrm{L}$, uric acid increased to $9.2 \mathrm{mg} / \mathrm{dL}$, phosphorus increased to $6.2 \mathrm{mg} / \mathrm{dL}$, ionized calcium dropped to $0.72 \mathrm{mmol} / \mathrm{L}$, and creatinine increased to $2.27 \mathrm{mg} /$ days (Table 1). A diagnosis of TLS was made, and the patient was given rasburicase and allopurinol for hyperuricemia, D50 and insulin for hyperkalemia, and nephrology was consulted for initiation of urgent hemodialysis. The electrolyte abnormalities resolved following hemodialysis, but the patient's kidney function remained poor, reaching a maximum creatinine level of $3.28 \mathrm{mg} / \mathrm{dL} 2$ days later. Calculated GFR had dropped over the course of the hospital stay from a baseline of $64 \mathrm{~mL} / \mathrm{min} / 1.73 \mathrm{~m}^{2}$ to $13 \mathrm{~mL} / \mathrm{min} / 1.73 \mathrm{~m}^{2}$. A family meeting was held, and the decision was made to place the patient on comfort care. She died 7 days following her chemotherapy. No follow-up imaging or CA 19-9 levels were obtained.

\section{Discussion}

TLS is the most common hematologic emergency consequence of chemotherapy, primarily seen in cases of hematologic malignancies treated with aggressive drug regimens. Patients with solid tumors have previously been found to only be at $1 \%$ risk of getting TLS [5]. Additionally, 
Table 1. Laboratory values after the first chemotherapy treatment

\begin{tabular}{llll}
\hline Lab parameter & Value & Lab parameter & Value \\
\hline $\begin{array}{l}\text { Basic metabolic panel } \\
\text { Sodium level }\end{array}$ & $125 \mathrm{mmol} / \mathrm{L}$ & $\begin{array}{c}\text { Complete blood count } \\
\text { Phite blood }\end{array}$ & $20.9 \times 10^{3}$ \\
Potassium level & $6.1 \mathrm{mmol} / \mathrm{L}$ & Red blood cell & $3.03 \times 10^{6}$ \\
Chloride & $96 \mathrm{mmol} / \mathrm{L}$ & Hemoglobin & $9.6 \mathrm{~g} / \mathrm{dL}$ \\
Bicarbonate & $14 \mathrm{mmol} / \mathrm{L}$ & Hematocrit & $27.9 \%$ \\
Anion gap & $15 \mathrm{mmol} / \mathrm{L}$ & MCV & $92.1 \mathrm{fL}$ \\
BUN & $44 \mathrm{mg} / \mathrm{dL}$ & MCHC & $34.2 \mathrm{~g} / \mathrm{dL}$ \\
Creatinine & $2.27 \mathrm{mg} / \mathrm{dL}$ & Platelet count & 495,000 \\
Glucose & $164 \mathrm{mg} / \mathrm{dL}$ & Liver function tests & \\
Calcium & $8.2 \mathrm{mg} / \mathrm{dL}$ & Aspartate transaminase & $48 \mathrm{U} / \mathrm{L}$ \\
Others & & Alanine transaminase & $30 \mathrm{U} / \mathrm{L}$ \\
Uric acid & $9.2 \mathrm{mg} / \mathrm{dL}$ & Alkaline phosphatase & $304 \mathrm{U} / \mathrm{L}$ \\
Lactic acid & $1.1 \mathrm{mmol} / \mathrm{L}$ & Total bilirubin & $0.2 \mathrm{mg} / \mathrm{dL}$ \\
Phosphorus & $6.2 \mathrm{mg} / \mathrm{dL}$ & Direct bilirubin & $<0.2 \mathrm{mg} / \mathrm{dL}$ \\
Ionized calcium & $0.72 \mathrm{mmol} / \mathrm{dL}$ & & \\
\hline
\end{tabular}

BUN, blood urea nitrogen; MCV, mean corpuscular volume; MCHC, mean corpuscular hemoglobin concentration.

there are specific risk factors which have been shown to correlate with TLS. These tumor risk factors include having high tumor proliferation rate, high sensitivity to chemotherapy, bulky disease $>10 \mathrm{~cm}$, and/or white blood cell count $>50,000$ and/or $\mathrm{LDH}>2$ times the upper limit of normal [6]. Increased risk also depends on whether or not there is other organ or bone marrow infiltration. Other clinical indications are an elevated uric acid level or phosphate levels, any prior exposure to nephrotoxic drugs, acidic urine and/or oliguria, and lastly dehydration [7]. Additionally, those with underlying impaired renal function prior to starting chemotherapy have also been found to have an increased risk for developing TLS [7].

Based on the above information, prior to receiving chemotherapy treatment, our patient had minimal signs to suggest a possible increased risk for development of TLS. At the time of diagnosis, primary tumor size was noted to be $3.5 \mathrm{~cm} \times 3.8 \mathrm{~cm} \times 3.2 \mathrm{~cm}$, located in the tail of the pancreas, which remained largely unchanged throughout the hospital course ( 3 weeks). Lab values were never suspicious for an elevated risk of TLS development. Known risk factors that were present in this patient were metastatic lesions, specifically involving the liver and the peritoneum, and impaired renal function due to history of renal cell carcinoma requiring a nephrectomy. At the time of treatment initiation, it was felt that the patient was at low risk for TLS.

\section{Conclusion}

While TLS can lead to death, the prognosis is typically excellent if caught early on in the course. This case demonstrates the need for high clinical suspicion when initiating any course of chemotherapy, even when the dosing is for palliation. It is particularly important to be acutely aware of electrolyte changes in the setting of bulky solid tumor such as pancreatic adenocarcinoma and other malignancies with a high burden of metastatic disease. Currently, 
guidelines for TLS prophylaxis are lacking. Further work is necessary to elucidate a truly beneficial risk stratification system to identify patients that would benefit from prophylaxis. This case highlights the importance of considering TLS prophylaxis in a patient with a highly aggressive, fast-growing tumor and widespread metastatic disease.

\section{Statement of Ethics}

All research was conducted ethically in accordance with the World Medical Association Declaration of Helsinki. The subject discussed in the above case report has given written informed consent to publish information regarding the case. All patient information has been de-identified.

\section{Conflict of Interest Statement}

The authors have no conflicts of interest to declare.

\section{Funding Sources}

No funding was utilized to carry out the above research.

\section{Author Contributions}

Paul Travers made substantial contributions to the conception or design of the work as well as acquisition, analysis, and interpretation of the data; drafted the work and revised it critically for important intellectual content; and agreed to be accountable for all aspects of the work in ensuring that questions related to the accuracy or integrity of any part of the work are appropriately investigated and resolved. Alexandra Goodman drafted the work and revised it critically for important intellectual content and agreed to be accountable for all aspects of the work in ensuring that questions related to the accuracy or integrity of any part of the work are appropriately investigated and resolved. Bernard Poiesz gave final approval of the version to be published.

\section{Data Availability Statement}

All data underlying the results are available as part of the article and no additional source data are required.

\section{References}

1 Howard SC, Jones DP, Pui CH. The tumor lysis syndrome. N Engl J Med. 2011;364(19):1844-54.

2 Krishnan SC, D'Silva CH, Al-Janadi A. Cetuximab-related tumor lysis syndrome in metastatic colon carcinoma. J Clin Oncol. 2008 May 10;26(14):2406-8.

3 Godoy H, Kesterson JP, Lele S. Tumor lysis syndrome associated with carboplatin and paclitaxel in a woman with recurrent endometrial cancer. Int J Gynaecol Obstet. 2010 Jun;109(3):254.

4 Cairo MS, Bishop M. Tumour lysis syndrome: new therapeutic strategies and classification. Br J Haematol. 2004 Oct; $127(1): 3-11$.

\section{Karger'}


5 Coiffier B, Altman A, Pui CH, Younes A, Cairo MS. Guidelines for the management of pediatric and adult tumor lysis syndrome: an evidence-based review. J Clin Oncol. 2008;26:2767.

6 Cairo MS, Bishop M. Tumour lysis syndrome: new therapeutic strategies and classification. Br J Haematol. 2004;127:3-11.

7 Hande KR, Garrow GC. Acute tumor lysis syndrome in patients with high-grade non-Hodgkin's lymphoma. Am J Med. 1993;94:133-9. 